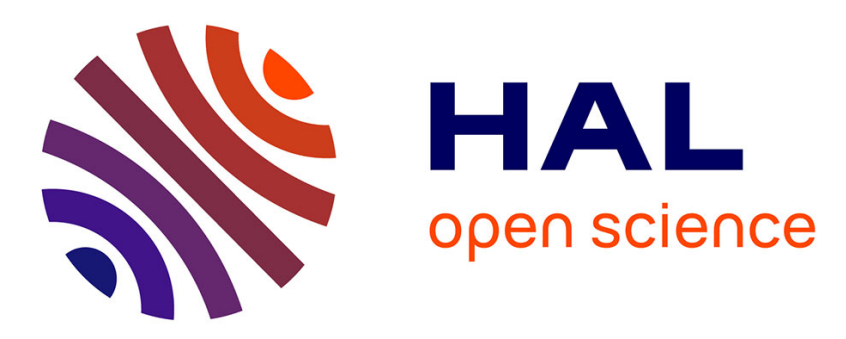

\title{
Interface energy in random systems
}

Bernard Derrida, J. Vannimenus

\section{- To cite this version:}

Bernard Derrida, J. Vannimenus. Interface energy in random systems. Physical Review B, 1983, 27

(7), pp.4401-4411. 10.1103/PhysRevB.27.4401 . hal-03285929

\section{HAL Id: hal-03285929 \\ https://hal.science/hal-03285929}

Submitted on 19 Jul 2021

HAL is a multi-disciplinary open access archive for the deposit and dissemination of scientific research documents, whether they are published or not. The documents may come from teaching and research institutions in France or abroad, or from public or private research centers.
L'archive ouverte pluridisciplinaire HAL, est destinée au dépôt et à la diffusion de documents scientifiques de niveau recherche, publiés ou non, émanant des établissements d'enseignement et de recherche français ou étrangers, des laboratoires publics ou privés. 


\title{
Interface energy in random systems
}

\author{
B. Derrida \\ Service de Physique Théorique, Centre d'Etudes Nucléaires de Saclay, \\ F-91191 Gif-sur-Yvette, France \\ J. Vannimenus \\ Groupe de Physique des Solides de l'Ecole Normale Supérieure, 24 rue Lhomond, \\ F-75231 Paris Cedex 05, France \\ (Received 17 May 1982; revised manuscript received 13 September 1982)
}

\begin{abstract}
We study the interface energy $\sigma$ as a function of disorder in two-dimensional Ising-type systems at $T=0$ for the percolation and frustration models. Our approach consists in calculating this energy for long strips of varying widths by a random sampling method, then extrapolating the results. In the weak-disorder limit, very wide strips may be studied and accurate values are obtained for the first-order correction to the interface energy in both models. Only moderate widths (up to $N=9-10$ ) can be studied in the general situation and we use finite-size scaling to analyze the data in the region of the threshold, where $\sigma$ vanishes with a critical exponent $v$. For percolation, we obtain $v / v=0.98 \pm 0.05$, where $v$ is the correlation-length exponent, in agreement with Deutscher and Rappaport's proposal that $v=v$ exactly. The analysis of the results is more difficult in the case of frustration, because size effects are important and the scaling region is not reached for $N=9$. Our data show that Monte Carlo results for $\sigma$ are unreliable and that much care is necessary to reach firm conclusions on the frustration threshold $x_{c}$ or the exponents $v$ and $v$.
\end{abstract}

\section{INTRODUCTION}

The notion of an interface energy in random systems arises in a number of contexts, e.g., the domain-wall energy in a dilute Ising-type ferromagnet $^{1}$ and the fracture energy in a solid containing a density of defects (when long-range bonding forces do not dominate). It is also directly related to the critical current density in models of dilute superconductors $^{2-4}$ and of superfluid flow in a porous medium, ${ }^{5}$ and it has been studied in systems with competing interactions, in connection with the spin-glass transition. $^{6-8}$ Many interesting questions remain to be answered. For instance, it proves difficult to obtain explicit results even for weakly disordered systems, whereas this is easy for the bulk free energy or magnetization. The origin of the difficulty is that an interface is a global object and this has deep consequences on its behavior. Also the interface energy is the difference between two bulk energies, and its numerical evaluation is often not very accurate, except in some simple situations. In particular, it is difficult to use Monte Carlo methods for frustrated systems, ${ }^{8}$ because one has to perform the difference between the results of two separate runs.

Here we propose an approach that relies on the transfer-matrix method for systems of finite width. This method has now been applied to a large num- ber of problems and in conjunction with finite-sizescaling ideas, it yields accurate results for critical properties with a reasonable amount of effort. ${ }^{9-12}$ Previous works focused mostly on such quantities as the correlation or connectivity length, the specific heat or the susceptibility, but the method may be generalized to many physical properties, once a suitable formulation has been found.

To illustrate the approach and make explicit calculations, we choose as representative interface the domain wall in an inhomogeneous magnetic system. We consider in detail two models on the square lattice at zero temperature: the bond-diluted ferromagnet (the percolation model), for which a lot of precise results are available, and the frustration model ${ }^{13}$ much studied in connection with spin-glasses, for which the situation is more controversial.

Two regimes are of particular interest in such systems. In the weak-disorder limit, only a very small fraction $x$ of the bonds are modified and it is plausible that the interface energy $\sigma$ varies linearly with $x$ :

$$
\sigma=\sigma_{0}(1-a x+\cdots) .
$$

This linear correction turns out to be correct, but in both models its calculation is more intricate than one would expect and is a challenging problem by itself. It is possible to give a formulation somewhat similar to the solid-on-solid model of crystal-growth 
theory, ${ }^{14}$ which reduces considerably the complexity of the calculations. Very wide strips (up to $10^{3}$ ) can thus be studied and the value of $d \sigma / d x$ for the twodimensional system is obtained through a direct extrapolation. It depends on the details of the model, but the formulation of the problem and the method used may be of general interest: The important point is that in spite of the weakness of the disorder, a simple effective-medium-type approximation does not seem feasible and the interface properties are intrinsic to the disordered system.

The second regime corresponds to the region of the threshold $x_{c}$ where $\sigma$ is expected to vanish with a characteristic exponent $v$ (we follow the notation introduced for the critical-current density ${ }^{2-4}$ ):

$$
\sigma \sim A\left(x_{c}-x\right)^{v} .
$$

For strips of finite width, the interface energy remains finite at $x_{c}$ and is still sizable in the critical region for the largest widths we can study $(\leq 10)$. A scaling analysis of the size dependence is then necessary to extract $v$ from our data. Such an analysis is easy in the case of percolation because the threshold is known exactly as well as the correlation-length exponent $v$ which plays a key role in the scaling equations. The result agrees with the proposal ${ }^{2,15}$ that in two dimensions $v=v$, which is also corroborated by Monte Carlo simulations ${ }^{3}$ and scaling studies on finite cells. ${ }^{4}$ In the case of frustration, the same type of analysis is less conclusive, partly because the threshold $x_{c}$ is not known accurately and the exponent $v$ has not been determined yet. Also, it suggests that size effects are much more important in frustrated systems than in their dilute counterparts.

This paper is organized as follows. In Sec. II the models studied are defined and several possible definitions of the interface energy are discussed. The transfer-matrix approach is explained and used to compute $\sigma$ analytically for the simplest cases. It is shown in the next section how the problem may be simplified in the weak-disorder limit. The coefficient $a$ of the linear term in Eq. (1) is calculated, exactly for narrow strips and by a random sampling method for large strips. Section IV is concerned with the critical region. We write the finite-size scaling equations for $\sigma$ and use them to analyze the data obtained on random samples. The results are discussed and compared with other approaches.

\section{DEFINITIONS AND PRINCIPLE OF THE METHOD}

\section{A. Definitions}

The systems considered are defined by Hamiltonians of the type

$$
\mathscr{H}=-\sum J_{i j} S_{i} S_{j},
$$

where $S_{i}$ are Ising spins on the sites of a square lattice. The nearest-neighbor interactions $J_{i j}$ are independent random variables; for the dilute ferromagnet their probability distribution is

$$
P\left(J_{i j}\right)=(1-p) \delta\left(J_{i j}\right)+p \delta\left(J_{i j}-J\right),
$$

while the frustration model corresponds to

$$
P\left(J_{i j}\right)=x \delta\left(J_{i j}+J\right)+(1-x) \delta\left(J_{i j}-J\right) .
$$

In a pure ferromagnet the interesting quantity is the temperature dependence of the interface free energy ${ }^{16,17}$ which may be defined in several ways that become equivalent for large systems. In the first definition [Fig. 1(a)], one fixes the orientation of the spins on two opposite sides of a sample of length $L$, and $\sigma$ is obtained from the difference between the free energy $E(+-)$ of the configuration where the spins of the two boundaries point in opposite directions and $E(++)$ of the configuration where they all point in the same direction:

$$
\sigma_{\mathrm{rc}}=[E(+-)-E(++)] / L,
$$

where rc stands for rigid boundary conditions. In the other standard definition, the boundary conditions (b.c.), are, respectively, periodic (pc) and antiperiodic [Fig. 1(b)]:

$$
\sigma_{\mathrm{pc}}=\left(E_{\mathrm{anti}}-E_{\mathrm{per}}\right) / L,
$$

which has usually the advantage to minimize size effects.

Here, for inhomogeneous systems, we fix $T=0$ and study the interface energy as a function of the (a)

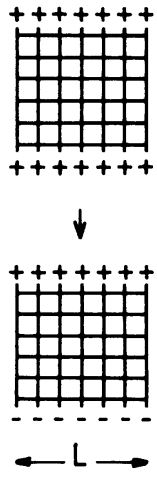

(b)

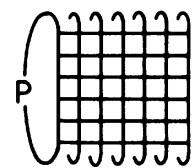

$\downarrow$

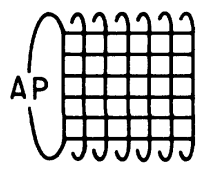

(c)

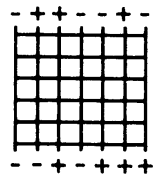

$\downarrow$

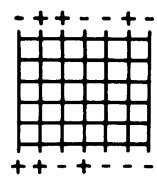

FIG. 1. Different types of boundary conditions (b.c.) that may be used to define the interface energy: (a) rigid b.c.; (b) periodic (P) and antiperiodic (AP) b.c.; (c) free b.c. The interface energy is given in all cases by the energy difference between the bottom and top configurations. 
concentrations $p$ or $x$. The preceding definitions, Eqs. (6) and (7), remain well suited to these systems when the interactions are positive or zero. When ferromagnetic and antiferromagnetic interactions coexist, a more general definition is in principle better. ${ }^{18}$ One starts from the configuration that minimizes the total energy with free boundary conditions at the surface, then reverses the spins on one edge while holding the other edge fixed [Fig. 1(c)]. The interface energy (at $T=0$ ) is equal to the energy increase between the initial state and the state that minimizes the energy with the new boundary conditions. For diluted ferromagnetic interactions, it is equivalent to the rigid b.c., but not for the frustration model. This general definition [Fig. 1(c)] is, however, not tractable by our methods, so we use the definitions (6) and (7) even for the frustration model. These should be adequate so long as long-range ferromagnetic order exists, but they may be problematic to study the existence of a spin-glass phase.

\section{B. The transfer-matrix method}

The transfer-matrix approach for disordered systems has been described in some detail in a previous paper ${ }^{19}$ where it was shown how the free energy of an infinite strip may in general be obtained from the limiting behavior of a product of transfer matrices. A simpler formulation can be given in the $T=0 \mathrm{lim}$ it. The Hamiltonian for a strip of width $N$ is of the form

$$
\mathscr{P}=\sum_{k}\left(h_{k}+g_{k, k+1}\right) .
$$

Here $h_{k}$ contains the interactions inside column $k$ :

$$
h_{k}=-\sum_{i=1}^{N} J((k, i),(k, i+1)) S_{k, i} S_{k, i+1},
$$

where $(k, i)$ denote the integer coordinates of a site, and $g_{k, k+1}$ the interactions between spins in the same row $i$ but in successive columns:

$$
g_{k, k+1}=-\sum_{i=1}^{N} J((k, i),(k+1, i)) S_{k, i} S_{k+1, i}
$$

Let us call $E_{L}(\mathscr{C})$ the ground-state energy of a semi-infinite strip going from $(-\infty)$ to column $L$ when the spins in the last column have a given configuration $(\mathscr{C})$. Recurrence relations exist between successive sets of $E_{L}$,

$$
\begin{aligned}
E_{L+1}(\mathscr{C})= & h_{L+1}(\mathscr{C})+\min _{\left(\mathscr{C}^{\prime}\right)} \\
& \times\left[E_{L}\left(\mathscr{C}^{\prime}\right)+g_{L, L+1}\left(\mathscr{C}, \mathscr{C}^{\prime}\right)\right] .
\end{aligned}
$$

The principle of our calculation is to generate the interactions at random according to the distributions
(4) or (5) and iterate relation (9) to obtain $E_{L}(\mathscr{C})$. After a large number of iterations, the average of $E_{L} / L$ over the distribution $P(J)$ is automatically performed, since (9) defines a Markov process with a finite number of states ${ }^{19}$ - every state corresponds to a choice of the (integer and bounded) differences $E_{L}(\mathscr{C})-E_{L}\left(\mathscr{C}^{\prime}\right)$ for all pairs $\left(\mathscr{C}, \mathscr{C}^{\prime}\right)$.

For a given width, the accuracy on the groundstate energy is only limited by the length of the strip studied (supposing a perfect random-number generator) and in practice, a length of $10^{4}$ provides satisfactory results. Moreoever, since we compute exactly the ground-state energy with both sets of boundary conditions on the same sample, the finite length is the only source of error in the interface energy. This is in contrast with conventional Monte Carlo simulations ${ }^{8}$ where it is not practical to obtain the true ground state for samples larger than about $20 \times 20$, and the accuracy on $\sigma$ is rather low because it is obtained as the difference between two energies calculated independently.

As a check on the present approach, we have computed the ground-state energy for the frustration model at $x=\frac{1}{2}$, with periodic b.c. A sizable parity effect is observed, with the even ( 2 to 8 ) and odd (3 to 9) strips forming two distinct groups. Both groups are well fitted by an expression

$$
E_{N} \sim E_{\infty}-A / N^{2},
$$

with two distinct constants $A_{\text {even }}$ and $A_{\text {odd }}$, but a unique extrapolated value $E=-1.403 \pm 0.003$, in excellent agreement with the results obtained by other approaches:

$$
\begin{aligned}
E & =-1.40 \pm 0.01 \\
& =-1.403
\end{aligned}
$$

as in Refs. 20 and 21, respectively.

\section{Analytic results}

For very narrow strips, it is possible to obtain analytically the stationary probabilities for all the states of the Markov process defined by relations (9). In percolation the uniform state is an obvious ground state for periodic b.c. and only the antiperiodic b.c. need be studied. One finds for a strip of width $N=2$,

$$
\frac{\sigma_{\mathrm{pc}}}{J}=2 p^{2}\left(\frac{1+p+2 p^{2}+p^{3}}{1+p+3 p^{3}}\right) .
$$

In the frustration case, both types of b.c. have to be studied and one finds for $N=2$, 


$$
\begin{aligned}
& \frac{E_{\mathrm{per}}}{J}=-\frac{1}{4}\left(\frac{10-3 z^{2}+8 z^{4}+z^{6}}{2-z^{2}+z^{4}}\right), \\
& \frac{E_{\mathrm{anti}}}{J}=-\frac{\left(5-z^{2}\right)}{4},
\end{aligned}
$$

with $z=(1-2 x)$. The formulas become rather long for $N=3$ :

$$
\frac{E_{\mathrm{per}}}{J}=-\frac{1}{3} \frac{N(z)}{D(z)},
$$

with

$$
\begin{aligned}
N(z)= & 26880\left(1-2 z+\frac{9}{4} z^{2}\right)-59392 z^{3}+64332 z^{4}-44488 z^{5}+22858 z^{6}-9104 z^{7}-1488 z^{8} \\
& +12625 z^{9}-11535 z^{10}+5856 z^{11}-1188 z^{12}-439 z^{13}+1353 z^{14}-1000 z^{15} \\
& +224 z^{16}+179 z^{17}-133 z^{18}+24 z^{19}+8 z^{20}-5 z^{21}+z^{22} \\
D(z)= & 6528\left(1-2 z+\frac{9}{4} z^{2}\right)-15040 z^{3}+15522 z^{4}-10492 z^{5}+5447 z^{6}-2609 z^{7}+130 z^{8} \\
& +2202 z^{9}-2149 z^{10}+1173 z^{11}-434 z^{12}+80 z^{13}+173 z^{14}-167 z^{15}+46 z^{16}+18 z^{17}-15 z^{18}+3 z^{19},
\end{aligned}
$$

and $E_{\text {anti }}$ is obtained by changing $z$ into $-z$ in these formulas. In general, the energy will be given by a rational expression but the calculations soon become intractable analytically. These exact results are nonetheless useful as checks on the numerical method presented above.

\section{INTERFACE IN WEAKLY DISORDERED SYSTEMS}

\section{A. Nature of the problem}

When a system is weakly disordered, it is often possible to relate its properties to those of the pure homogeneous system (assumed to be completely known). For instance, one can obtain the first term in the expansion of the free energy of the dilute ferromagnet, ${ }^{22}$ and of its critical temperature (supposing the nature of the singularity is not modified by weak disorder). Another example is given by the ground-state energy and zero-temperature magnetization of the frustration model, ${ }^{23}$ for $x<1$. This is achieved by noting that when the inhomogeneities are very far apart they do not interact and their contributions to extensive quantities such as the free energy or the magnetization are simply additive.

$A$ priori, one might think that a similar approach is possible for the interface energy, and indeed an expansion of this type has been attempted ${ }^{6}$ - but it is incorrect. The reason is that the interface is a nonlocal object: Its shape and energy depend on the precise arrangement of the impurities, even if they are very distant. In fact, as we shall see, the interface adopts shapes that are unfavorable locally in order to minimize its total energy. This aspect has been overlooked in previous work, ${ }^{6}$ leading to an overestimation of this energy, and we present here a correct approach to the problem.

\section{B. Shape of the interface}

Let us consider for definiteness the case of a frustrated strip of width $N$, with rigid-boundary conditions. When $x<1$, the $(-J)$ bonds lie far apart and the unique ground state for $(++)$ b.c. is the pure ferromagnetic state. For $(+-)$ b.c. we first remark that it is never favorable to flip an isolated spin or cluster of spins, and so any configuration of minimum energy possesses a unique continuous boundary between $(+)$ and $(-)$ spins. It is then meaningful in this limit $x<1$ to speak of the interface as a physical object, not just the interface energy.

At low temperatures $T$ the interface will show thermally activated steps. ${ }^{14}$ However, at low enough $T$, the average distance $1(T)$ between such steps is much larger than the average distance between $(-J)$ bonds, if $\exp (-2 J / T) \ll x$. It is important to notice that the limits $T \rightarrow 0$ and $x \rightarrow 0$ do not commute: Here we study the case where thermally activated steps between two impurities are negligible. The other limit would be the situation where $T \rightarrow 0$ and $x \rightarrow 0$, with the constraint that the average distance between impurities is so large that there are many thermally activated steps between any pair of impurities.

A second important remark is that there is at most one negative bond per column, provided $N x \ll 1$. The interface may lower its total energy by crossing that bond, if this does not cost too much lateral displacement energy, or it may ignore the negative bond: The choice depends on what is coming next, but in any case overhangs are never favorable in the dilute limit (Fig. 2).

As a consequence, the possible ground states of the strip may be characterized by specifying the "channel" in which the interface lies at every 


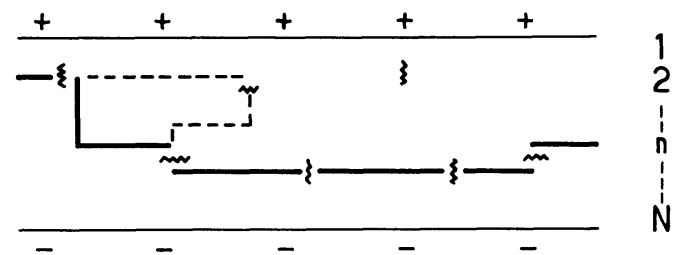

FIG. 2. Interface (heavy line) on a strip in the weakdisorder regime. Only the impurity bonds (wiggly lines) are drawn: They lie far apart and "overhangs" such as shown by the dotted line are never favorable energetically. Channel numbering is shown on the right: Note that for rigid b.c. as shown here there are $N-1$ rows of spins, in addition to the fixed boundary spins.

column. These channels separate two neighboring spin rows; there are $N$ possibilities instead of the $2^{N}$ configurations appearing in Eq. (9).

\section{Recurrence relations}

Consider the semi-infinite strip from $(-\infty)$ to column $L$. If the interface is constrained to lie in channel $n$ at column $L$, its energy $E_{n}$ depends on all the negative bonds on the left. The differences $\left(E_{n+1}-E_{n}\right)$ are equal either to $2 J$ or $-2 J$ and it is convenient to introduce the function

$$
f_{L}(n)=L-E_{n} / 2 J \text {, }
$$

which may always be represented by a broken line of slope \pm 1 (Fig. 3). All the needed information is contained in the large- $L$ behavior of this function.

How is $f_{L}(n)$ modified when the $2 N-1$ interactions of the $(L+1)$ th column (for rigid b.c.) are added to the strip? One has just to write Eq. (9) in this new notation. At first order in $x$, these interactions are all positive with probability

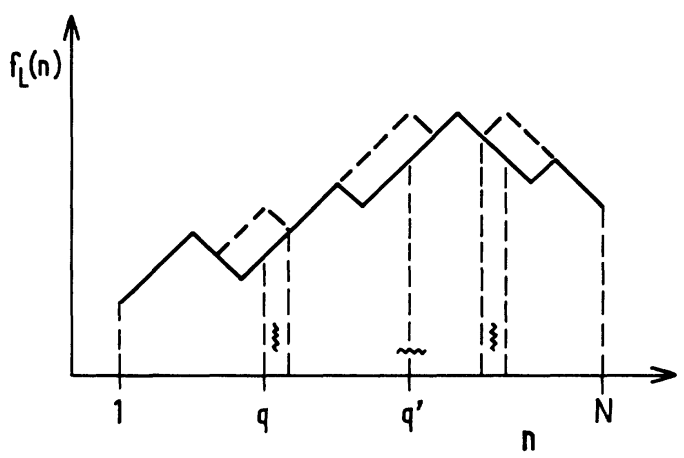

FIG. 3. Diagram of the function $f_{L}(n)$ representing the energy of the interface if it is constrained to lie in channel $n$ at column $L$. The action on $f_{L}(n)$ of negative bonds (wiggly lines) is depicted by the dotted lines: The bond between channels $q$ and $(q+1)$ lies parallel to the strip, the one at $q^{\prime}$ is perpendicular to the strip.
[ $1-(2 N-1) x]$, while one of them is negative, all the others being positive, with probability $x$. Cases with more than one negative bond in the same column would only contribute at order $x^{2}$ and thus can be forgotten here.

Three situations have to be distinguished.

(1) All interactions positive:

$$
f_{L+1}(n)=f_{L}(n) \text {, for all } n \text {. }
$$

(2) One negative bond, lying orthogonally to the strip on channel $q$ ( $N$ possible positions):

$f_{L+1}(q)=f_{L}(q)+2$,

$f_{L+1}(n)=\max \left[f_{L}(n), f_{L}(q)+2-|n-q|\right], n \neq q$.

(3) One negative bond, parallel to the strip, between channels $q$ and $q+1$ ( $N-1$ positions). Here, two subcases arise:

(i) if $f_{L}(q)>f_{L}(q+1)$ :

$f_{L+1}(n)=\left\{\begin{array}{l}f_{L}(n), n \leq q \\ \max \left[f_{L}(n), f_{L}(q)+2+q-n\right], \\ n \geq q+1 .\end{array}\right.$

(ii) if $f_{L}(q)<f_{L}(q+1)$ :

$f_{L+1}(n)=\left\{\begin{array}{l}\max \left[f_{L}(n), f_{L}(q)+2+n-q\right], n \leq q \\ f_{L}(n), \quad n \geq q+1 .\end{array}\right.$

The function $f_{L}(n)$ has $2^{N-1}$ possible shapes (not taking into account symmetries) and Eqs. (13) tell us how these shapes transform into one another. From these relations the stationary probabilities $p_{k}$ of all the different shapes may be computed. The interface energy per unit length is then given by

$$
\frac{\sigma_{N}}{2 J}=1-\sum_{k} p_{k}\left\langle f_{L+1}(n)-f_{L}(n)\right\rangle_{k},
$$

for any value of $n$ [because the result (14) is independent of $n$ ]. In this equation the angular brackets with subscript $k$ mean the average for shape $k$ over the configuration of the $2 N-1$ bonds in column $L+1$.

The only nonzero contributions to the sum in (14) come from the configurations with one negative bond, which have probability $x$. Writing that, for a strip of width $N, \sigma_{N}$ has a weak-disorder expansion of the form

$$
\sigma_{N}=2 J\left(1-a_{N} x+\cdots\right),
$$


TABLE I. Exact expressions for the linear term in the dependence of the interface energy with respect to disorder. For periodic boundary conditions and $N=2,3$, the results agree with the linear term in the expansion of the general formulas (11) and (12) given in Sec. II.

\begin{tabular}{ccccc}
\hline \hline $\begin{array}{c}\text { Strip width } \\
\text { (number of } \\
\text { channels) }\end{array}$ & Rigid b.c. & Periodic b.c. & Rigid b.c. & Periodic b.c. \\
\hline 2 & 4 & 5 & $3 / 2$ & $8 / 5$ \\
3 & $58 / 11$ & $249 / 38$ & $2013 / 1130$ & $25 / 13$ \\
4 & $672 / 109$ & $78 / 11$ & & $13658 / 6413$ \\
6 & & $3817 / 466$ & & \\
8 & & $8.86375 \ldots$ & & \\
\hline \hline
\end{tabular}

one obtains an expression for the coefficient $a_{N}$ by identifying (14) and (15). The same general approach works for percolation also, with the difference that $f_{L}(n+1)-f_{L}(n)$ may take the values 1,0 , and -1 .

\section{Results}

The calculations outlined above have been carried out analytically for small widths and the results obtained in this way are given in Table I. The periodic conditions are easier because the higher symmetry reduces the number of shapes to consider.

For larger widths we have studied Eqs. (13) numerically by generating random samples. Only columns with one negative bond have to be generated in the present approach, which is therefore much more efficient for weak disorder than the general method of Sec. II. It is possible to study very large widths $\left(N<10^{3}\right)$ for long strips (typically 5000N) even on a Digital Equipment Corporation PDP11/03 minicomputer, and the errors due to the finite length become very small for such large systems.

The reason to push the calculations very far is ap-

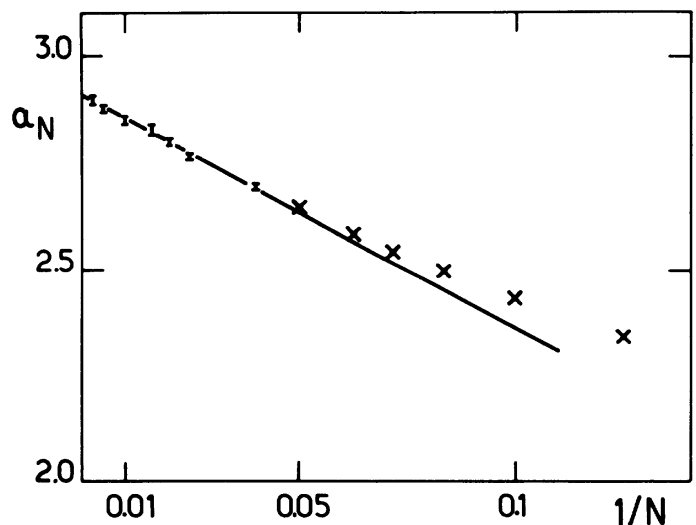

FIG. 4. Plot of $a_{N}=(2 J)^{-1} d \sigma_{N} / d p$ for $(1-p)<1$ in the percolation model $v s$ the inverse of the strip width (rigid b.c. are used). The bars indicate statistical fluctuations. For large $N$ the results are well approximated by the straight line, of equation $a_{N}=2.91-5.4 / N$. parent from the results displayed in Figs. 4 and 5. For percolation the size effects are not strong and moderate widths $(\sim 20)$ are sufficient to obtain a good estimate of $d \sigma / d p$; a linear extrapolation versus $1 / N$ fits the results very well. The data for frustration (Fig. 5) show on the contrary a significant upward curvature on a similar plot and the convergence law was not clear from moderate sizes. A variation with $N^{-1 / 2}$ seemed possible, or even that $a_{N}$ did not converge to a finite limit. The large-size results appear to rule out these possibilities and favor a simple $1 / N$ dependence [if $a_{N}$ is plotted versus $(\ln N) / N$, for example, the curvature is reversed].

The final extrapolated results read as follows:

$$
a=\lim _{N \rightarrow \infty} a_{N}=\left\{\begin{array}{c}
2.91 \pm 0.02 \\
11.63 \pm 0.05
\end{array}\right.
$$

in the percolation and frustration models, respectively. Several remarks can be made on these results.

(1) The value for frustration is nearly twice as

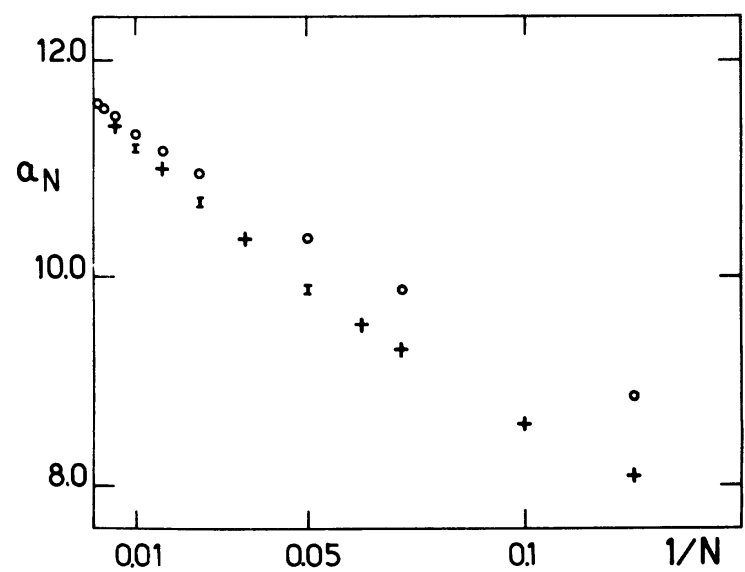

FIG. 5. Plot of $a_{N}=-(2 J)^{-1} d \sigma_{N} / d x$ for $x \ll 1$ in the frustration model vs $1 / N$. The crosses $(+)$ denote rigid b.c., the open circles $(O)$ periodic b.c. The bars indicate typical statistical fluctuations. Note the fourfold difference in vertical scale with Fig. 6. 
large as the value $a=6$ quoted in previous work. ${ }^{6}$ The former value had been obtained by considering the local shape modifications of a straight interface that take advantage of the inhomogeneities (or at least cost no energy). The large difference implies that the interface can lower considerably its energy by adopting locally unfavorable shapes.

(2) The slow convergence as a function of size (power law rather than exponential) implies that the interface is very delocalized and often comes into contact with the edges of the strip. The characterization of its "roughness" is an interesting problem which deserves more investigation.

(3) The approach followed here is justified only under the condition $N x \ll 1$. It is not obvious $a$ priori that higher-order terms in the expansion of $\sigma_{N}$ (Eq. 15) do not diverge when $N \rightarrow \infty$, preventing the expansion from having a well-behaved limit. This apparently does not happen, if we judge from the good agreement between the linear expansion and the numerical results obtained at concentrations of a few percent (presented below).

\section{CRITICAL REGIME}

\section{A. Finite-size scaling}

For an arbitrary concentration of impurity bonds, the size of the strips we can study with reasonable accuracy is rather limited. A direct extrapolation of the results as in the weak-disorder regime is not reliable in these conditions. However, there exists a more sophisticated method to analyze the results obtained on systems of finite size such as strips, when one is in the vicinity of a critical point. The finitesize scaling hypothesis ${ }^{24}$ relates the behavior of a physical property in a finite system and its singularity in the infinite system. Explicitly, if the interface energy for the two-dimensional system behaves as

$$
\sigma \sim A\left(x_{c}-x\right)^{v},
$$

this singularity is smeared out on a strip of width $N$ and for sufficiently large $N$ the interface energy is of the form

$$
\sigma_{N} \sim N^{-v / v} f\left[\left(x_{c}-x\right) N^{1 / v}\right] .
$$

Here $v$ is the critical exponent associated with the divergence of the correlation length and the scaling function $f(z)$ is regular near the origin. This method of analysis has been applied to many problems by now and was found to give good results for moderate strip widths for percolation. . $^{11,12}$

Our approach differs in an important way, however, from these works and most other previous work with the strip method: $\sigma_{N}$ is not known with arbitrary accuracy, because of the finite length of the strips, and this makes the use of Eq. (18) less straightforward. In particular, differentiation of (18) with respect to $x$ is not accurate enough to be of practical use. The situation is similar to the problem of size effects in standard Monte Carlo simulations and it is necessary to discuss how the data may be analyzed using the information available for a given problem.

\section{B. The percolation model}

The interface energy in the dilute Ising model at $T=0$ can be given a simple interpretation in terms of percolation concepts: It is equal to the minimum number of bonds one has to cut in order to disconnect the percolating cluster (above the percolation threshold), or equivalently to the maximum number of distinct independent paths one can draw that cross this cluster simultaneously. ${ }^{3,15}$

This interpretation indicates that the parameters entering the finite-size scaling equation (18) are those relevant to percolation: the threshold $p_{c}=\frac{1}{4^{2}}$ and the connectedness-length exponent $v=\frac{4}{3}$. Moreover, arguments based on a simple picture of the percolation cluster have been given ${ }^{2,15}$ that one has exactly

$$
v=v
$$

in two dimensions, as for the corresponding singularity of the interface free energy at $T_{c}$ in the pure Ising model. ${ }^{17}$ This prediction is well supported by Monte Carlo simulations. ${ }^{3}$

As a consequence of these remarks and of Eq. (18), the interface energy is expected to behave as

$$
\sigma_{N} \sim N^{-1} f\left[\left(p-p_{c}\right) N^{3 / 4}\right],
$$

for large $N$ and $\left(p-p_{c}\right) \ll 1$. A first implication of Eq. (20) is that at the threshold

$$
N \sigma_{N} \rightarrow \text { const }\left(p=p_{c}=0.5\right) \text {. }
$$

This can be checked directly on our data, but it is more instructive to plot $\ln \sigma_{N}$ vs $\ln N$ (Fig. 6). For $p>p_{c}, \sigma_{N}$ converges to a finite value for large $N$ and the graph shows an upward curvature while for $p<p_{c}$ it decreases with $N$ faster than a power law and the curvature is downward. Right at the threshold, $\ln \sigma_{N}$ decreases linearly with $\ln N$ and the slope gives the ratio $(v / v)$.

For a given computing time, one can either take averages over many short strips or study fewer but longer samples. We have chosen the second solution, and checked on five samples typically that the fluctuations were indeed small. The data in Fig. 6 bear on samples of length $10^{4}(2<N<7)$ or $2.10^{3}$ $(N=8,9)$, with periodic and antiperiodic boundary conditions. 


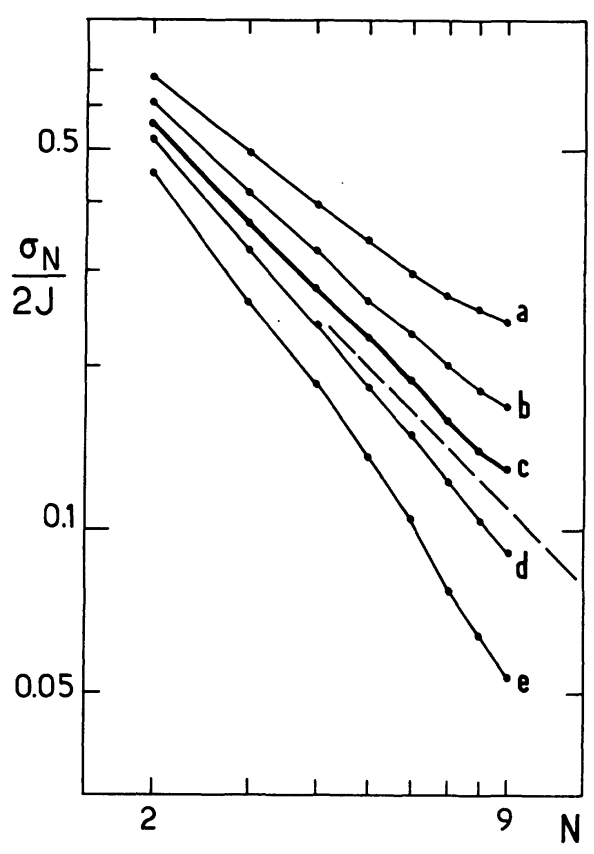

FIG. 6. Doubly logarithmic plot of $\sigma_{N}$ vs $N$ in the region of the percolation threshold: (a) $p=0.55$; (b) $=0.52$; (c) $=p_{c}=0.5$ (bold line); (d) $=0.48$; (e) $=0.45$. (Only the heavy dots have physical significance). The dotted line indicates the slope corresponding to a critical exponent $v=v$.

The agreement of the data with Eq. (21) is quite good. It can still be improved by fitting the raw data with a quadratic polynomial in $p$ for given $N$ in order to smooth the residual fluctuations. A graphical measure of the slope for $p=\frac{1}{2}$ gives

$$
v / v=0.98 \pm 0.05 \text {. }
$$

If $p_{c}$ were not known exactly it could be determined from the graph in Fig. 6, just by looking for the value of $p$ such that $\ln \sigma_{N}$ depends linearly on $\ln N$. A range of values is in fact found, because the range of $N$ is limited, and they correspond to different possible values of the slope, i.e., $v / v$. In any case, the overall curvature of the graphs changes sign between $p=0.45$ and $p=0.55$, showing clearly that a transition occurs in between.

Another way to analyze the data consists in plotting $N \sigma_{N}$ as a function of $z=\left(p-p_{c}\right) N^{3 / 4}$ to check directly the scaling prediction in Eq. (17). This is done in Fig. 7 and the results are well-fitted by a single scaling function, even for $N$ as small as 3 .

An interesting remark is that the quality of the fit is much more sensitive to the value of the ratio $v / v$ than to the value of $v$ itself. Also, if we let $p_{c}$ be an adjustable parameter, rather good fits can be obtained with other sets of values, e.g., $p_{c}=0.46$,

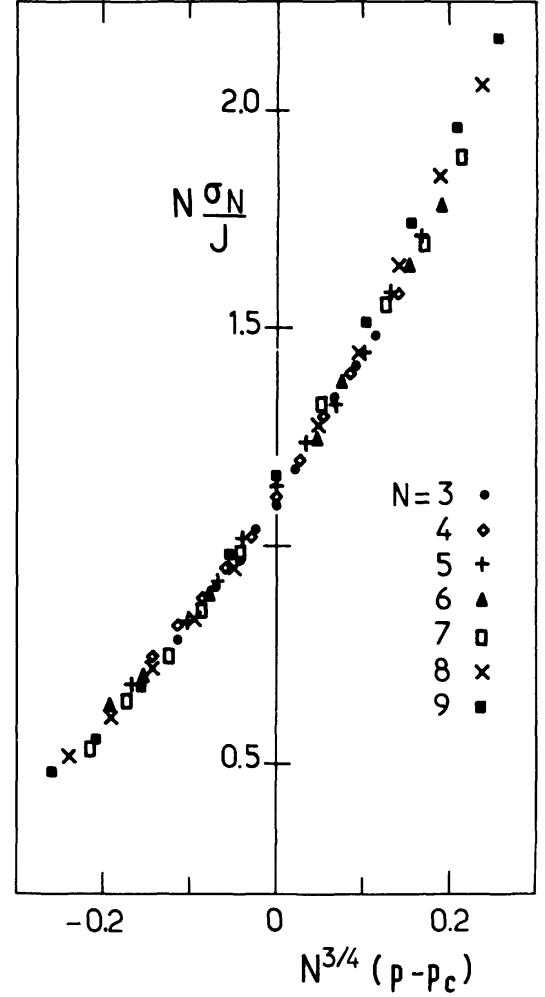

FIG. 7. Universal scaling function for the interface energy $\sigma_{N}$ near the percolation threshold. Some points are omitted to avoid excessive overlapping of the symbols.

$v / v=0.7$, and $v=\frac{4}{3}$. This shows clearly that $N=9$ is not a sufficient size to determine all three parameters without any extra information.

\section{The frustration model}

\section{Numerical results}

The results presented previously for percolation are satisfactory enough to try and apply the same approach to the more difficult frustration problem. We have generated random samples of length $10^{4}$ (for $2<N<7$ ) or $2.10^{3}$ (for $8<N<10$ ), and obtained the corresponding interface energy $\sigma_{N}$ for both periodic and rigid boundary conditions.

In Fig. 8 we show the results with periodic b.c. The comparison of the numerical results for $N=2$ and 3 with the full curves, which represent the exact expressions given in Eqs. (11) and (12), show that the statistical errors are very small and provide a useful check on our programs.

Figure (9) displays the results for rigid b.c., which have a more regular dependence on $N$. Intermediate points were also studied for $0.085<x<0.145$ but they are not shown for clarity.

Several immediate remarks may be made about Figs. 8 and 9. 


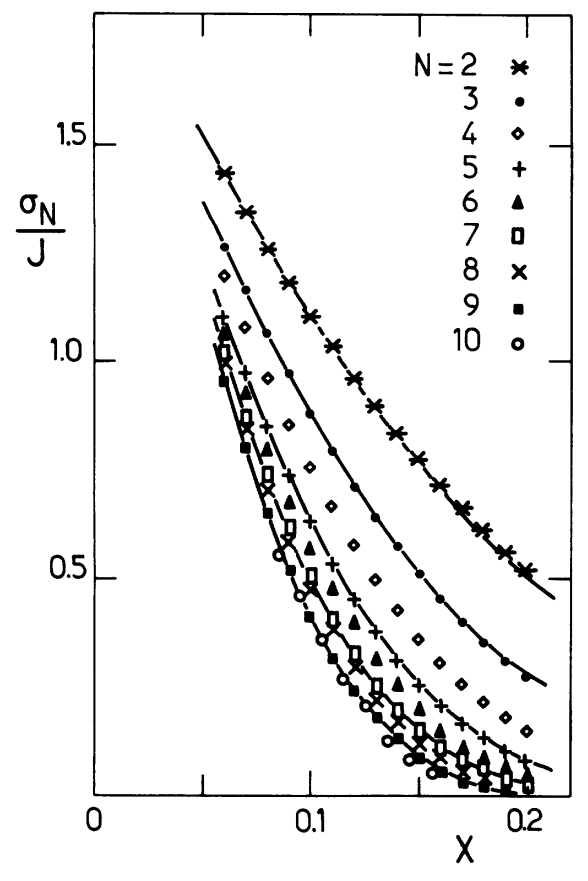

FIG. 8. Interface energy vs the concentration $x$ of negative bonds for the frustration model (periodic b.c.). The curves represent the exact results for $N=2$ and 3 (the other lines are just guides to the eye for $N=5,7,9)$.

(1) Both types of boundary conditions give very similar results. The periodic b.c. are somewhat less convenient to analyze, due to a marked parity effect.

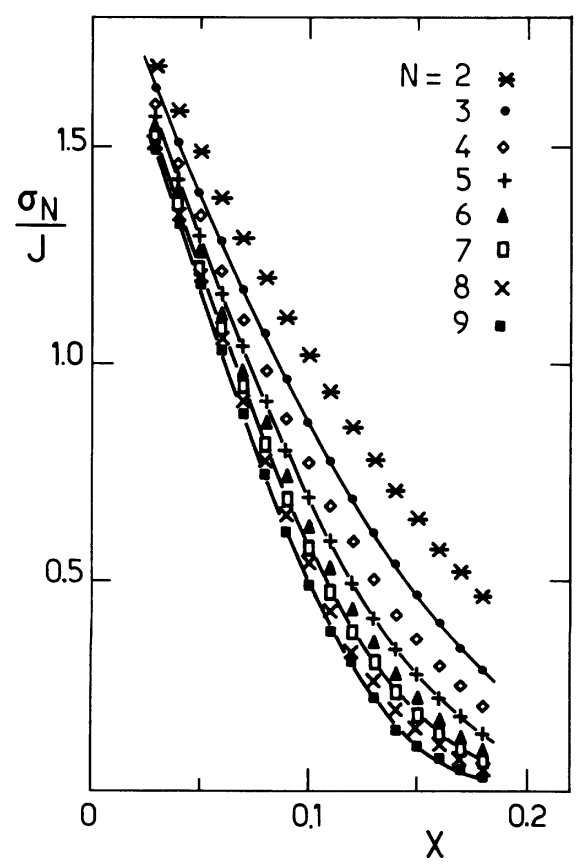

FIG. 9. Similar plot, with rigid b.c.
(2) For small values of $x(x<0.06), \sigma_{N}$ appears to converge towards a finite value when $N$ increases. The data are also consistent with the linear approximation of the weak-disorder regime, Eq. (15), suggesting that the terms quadratic in $x$ are well behaved.

(3) For large values of $x(x>0.14)$, there is no doubt that $\sigma_{N}$ extrapolates to 0 .

(4) In the intermediate region the convergence of $\sigma_{N}$ is quite slow and it is difficult to perform accurate extrapolations to determine the threshold $x_{c}$. However, the value obtained on a strip of width $N=9$ for $x=0.10, \sigma \simeq 0.4 J$ (see Fig. 8 ) is already well below the corresponding Monte Carlo estimate ${ }^{8}$ $\sigma=0.6 \mathrm{~J}$, obtained on $(55 \times 55)$ samples. Any reasonable extrapolation will necessarily yield a much lower value; this shows that the standard Monte Carlo approach is not adequate to study the interface, presumably because the true ground states are not obtained.

\section{Analysis}

We can apply for the data of Fig. 9 the same type of analysis as for percolation and, for instance, fit them by a scaling function of the form given in Eq. (18). Unfortunately, much less is known on the frustration model than on the percolation model. Even the value of the threshold $x_{c}$ is not known accurately and there is a rather wide spreading in the results obtained by different approaches, ${ }^{6,20,25,26}$ with the most likely value of $x_{c}$ in the range $0.10-0.15$. As for critical exponents, none have been determined so far. A precise analysis of the size dependence of $\sigma_{N}$ allows us in principle to determine the three parameters $x_{c}, v$, and $v$ simultaneously, but as discussed above, the maximum width reached in practice $(N=9$ or 10$)$ is not sufficient in the absence of extra information. The use of larger sizes is difficult because for a given accuracy the computing time increases exponentially with $N$.

The other less ambitious approach consists in plotting $\ln \sigma_{N}$ vs $\ln N$, in order to determine $x_{c}$ as the point where this curve changes its convexity. The corresponding curves are drawn in Fig. 10, for rigid b.c.

Two distinct regimes are apparent from the behavior of this set of curves:

(i) For $x>0.13$, the concavity of the curves is clearly downwards, meaning that $\sigma_{N}$ vanishes faster than a power law, so $x_{c}<0.13$.

(ii) For $x<0.13$, the curves show no clear cut curvature and are roughly linear for $4<N<9$, if the statistical errors are taken into account.

Taken literally, this result would mean that the 
whole region $x<0.13$ is in a sense critical, but we are reluctant to believe that $\sigma$ vanishes and longrange ferromagnetic order does not exist for $x$ as small as 0.03 . The most reasonable interpretation is that size effects are much more important here than for the percolation problem and that, for small $x$, $\ln \sigma_{N}$ will eventually curve upwards and saturate at a nonzero value for $N \gg 10$. An indication that this is indeed happening may be obtained using the weak-disorder expansion studied above. Approximating $\sigma_{N}$ by its first-order expansion $2\left(1-\alpha_{N} x\right)$, with $\alpha_{N}$ computed for rigid boundary conditions, it is found that for $x=0.03$, for example, $\ln \sigma_{N}$ is nearly linear in $\ln N$ for $N=4$ to 16 and converges relatively slowly to its limiting value. The "experimental" data for $\sigma_{N}$ lie very close to this first-order approximation for $5<N<9$ and it is natural to assume that they behave similarly for larger $N$. This analysis gives strong indication that the numerical studies performed on rather small frustrated systems (typically $20 \times 20$ ) may suffer from similar important size effects.

\section{Discussion}

It is not possible using merely the data shown in Fig. 10 to give a reliable estimate of $x_{c}$, but if $x_{c}$ is

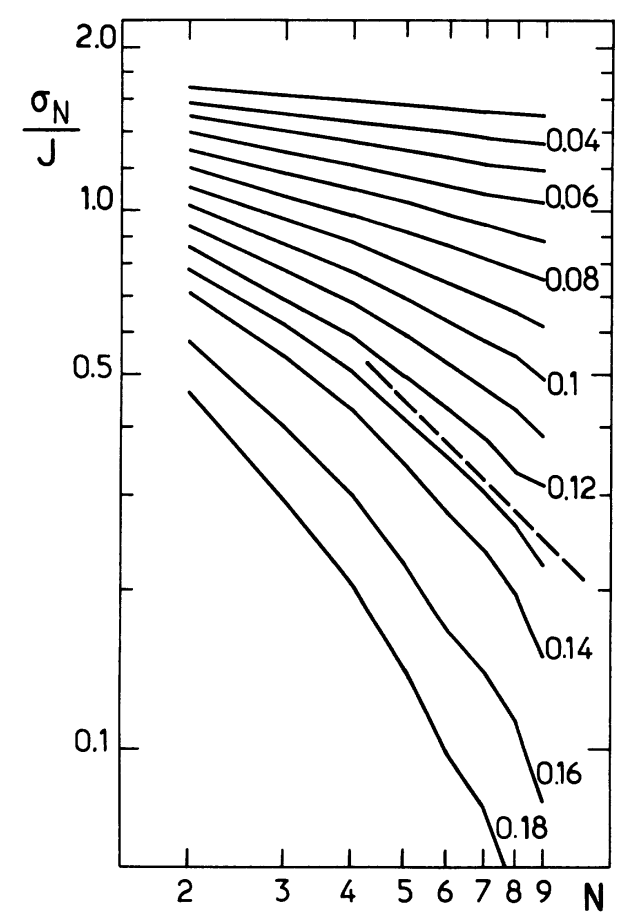

FIG. 10. Doubly logarithmic plot of $\sigma_{N}$ vs the strip width $N$, for $x$ varying from 0.3 to 0.18 (rigid b.c.). The dotted line indicates the asymptotic slope if the exponent $v=v$. determined by another method the slope of the curve corresponding to $x=x_{c}$ yields directly the ratio $v / v$, according to Eq. (18). Conversely, if one conjectures that $v=v$ as for the percolation problem, then the threshold is found to be $x_{c} \simeq 0.12 \pm 0.005$, a value in agreement with the results obtained by Morgenstern and Binder ${ }^{20}$ using the transfer-matrix method. A fit of $\sigma_{N}$ by a universal scaling function then gives $v=1.1 \pm 0.1$ as the most probable estimate.

The arguments usually given in support of the simple result $v=v$ in the percolation problem are based on pictures of the infinite percolating cluster and cannot be transposed immediately to the frustration problem. The basic idea is that the correlation (or connectivity) length is the only characteristic length near the threshold and that it governs also the width of the interface, as is the case for the liquid-vapor interface, for instance. ${ }^{17,27}$ For spinglasses and frustrated systems, experience has taught us to be cautious, and it is not clear that a single length characterizes the different types of correlations.

\section{CONCLUDING REMARKS}

In view of our results, it is an open and challenging question how the interface-energy singularity is related to the correlation length in the frustration problem. In their present state, our data provide a constraint on the values of the quantities $x_{c}, v$, and $v$, and progress for one of these would allow an easier determination of the others.

We wish to stress again the difference in spirit between the present approach, and the work of Morgenstern and Binder ${ }^{20}$ who have also studied disordered systems using a transfer-matrix formulation. These authors obtain the exact partition function of finite samples, up to $18 \times 18$, then study typically 25 realizations in order to perform averages over the disorder. In the present approach, no average has to be made; it suffices to let the length of the strips go to infinity. In practice very long strips can be studied and the statistical fluctuations are kept very small. This is an important advantage, both conceptually and practically, particularly near a transition point where fluctuations are likely to be important in the standard approach.

\section{ACKNOWLEDGMENTS}

We thank Professor J. L. Lebowitz for his hospitality at the Courant Institute, where part of this work was started by one of us (B.D), and R. B. Pearson for sharing his expertise in random-number generators. We are grateful to $\mathbf{R}$. Rammal for discussions of the interface problem and a critical reading of the manuscript. 
1This quantity is different from the stiffness constant of Heisenberg-type ferromagnets, which is related to the conductivity of a random resistor network. See S. Kirkpatrick, Rev. Mod. Phys. 45, 570 (1973).

${ }^{2}$ G. Deutscher and M. L. Rappaport, J. Phys. (Paris) Lett. 40, L219 (1979).

${ }^{3}$ S. Kirkpatrick, in Inhomogeneous Superconductors1979 (Berkeley Springs, West Virginia), Proceedings of the Conference on Inhomogeneous Superconductors, edited by D. U. Gubser, T. L. Francavilla, S. A. Wolf, and J. R. Leibowitz (AIP, New York, 1979), p. 79.

${ }^{4}$ C. J. Lobb and D. J. Frank, in Inhomogeneous Superconductors-1979 (Berkeley Springs, West Virginia), Proceedings of the Conference on Inhomogeneous Superconductors, edited by D. U. Gubser, T. L. Francavilla, S. A. Wolf, and J. R. Leibowitz (AIP, New York, 1979), p. 308.

${ }^{5}$ D. A. Huse and R. A. Guyer, Phys. Rev. Lett. 43,1163 (1979).

6J. Vannimenus and G. Toulouse, J. Phys. C $\underline{10}$, L537 (1977).

${ }^{7}$ P. Reed, M. A. Moore, and A. J. Bray, J. Phys. C 11 , L139 (1978).

${ }^{8}$ N. K. Jaggi, J. Phys. C $\underline{13}$, L177 (1980).

${ }^{9}$ M. P. Nightingale, Physica (Utrecht) $83 \mathrm{~A}, 561$ (1976).

${ }^{10}$ L. Sneddon, J. Phys. C 11, 2823 (1978); 12, 3051 (1979).

${ }^{11}$ B. Derrida and J. Vannimenus, J. Phys. (Paris) Lett. 41, L473 (1980); B. Derrida and L. de Seze, J. Phys. (Paris) $\underline{43}, 475$ (1982).

${ }^{12} \mathrm{~W}$. Kinzel and J. M. Yeomans, J. Phys. A $\underline{14}$, L163 (1981).

${ }^{13}$ G. Toulouse, Commun. Phys. $\underline{2}, 115$ (1977).
${ }^{14}$ For a recent review, see J. D. Weeks, in Ordering in Strongly Fluctuating Condensed Matter Systems, edited by T. Riste (Plenum, New York, 1980).

${ }^{15}$ M. J. de Oliveira, J. Phys. C 14, L317 (1981).

16P. G. Watson, in Phase Transitions and Critical Phenomena, edited by C. Domb and M. S. Green (Academic, New York, 1972), Vol. 2, p. 101.

${ }^{17} \mathrm{~A}$. Widom, in Phase Transitions and Critical Phenomena, Ref. 16, p. 79.

${ }^{18}$ B. Derrida, J. M. Maillard, J. Vannimenus, and S. Kirkpatrick, J. Phys. (Paris) Lett. 39, L465 (1978).

${ }^{19}$ B. Derrida, J. Vannimenus, and Y. Pomeau, J. Phys. C 11,4749 (1978).

${ }^{20}$ I. Morgenstern and K. Binder, Phys. Rev. B 22, 288 (1980).

${ }^{21}$ I. Bieche, R. Maynard, R. Rammal, and J. P. Uhry, J. Phys. A 13, 2253 (1980).

22M. F. Thorpe, J. Phys. C 11, 2983 (1978); E. Domany, ibid. 11, L337 (1978).

${ }^{23}$ G. Grinstein, C. Jayaprakash, and M. Wortis, Phys. Rev. B 19, 260 (1979); I. F. Lyuksyutov, Zh. Eksp. Teor. Fiz. 75, 1935 (1978) [Sov. Phys._JETP 4, 975 (1978)].

${ }^{24}$ M. E. Fisher, J. Phys. Soc. Jpn. Suppl. 26, 87 (1969); E. Brezin, J. Phys. (Paris) 43, 15 (1982).

${ }^{25}$ F. Baharona, R. Maynard, R. Rammal, and J. P. Uhry, J. Phys. A 15, 673 (1982); R. Maynard and R. Rammal, J. Phys. (Paris) Lett. 43, L347 (1982).

26J. R. L. de Almeida, I. Fittipaldi, and F. C. Sá Barreto, J. Phys. C 14 , L403 (1981).

${ }^{27}$ D. J. Wallace and R. K. P. Zia, Phys. Rev. Lett. 43, 808 (1979). 